\title{
QUANTUM SIZE EFFECTS IN ULTRATHIN LAYERED MAGNETIC SYSTEMS
}

\author{
P. BRUNo
}

Institut d'Électronique Fondamentale, CNRS URA 22

Bâtiment 220, Université Paris-Sud, 91405 Orsay, France

The effect of electron confinement in ultrathin layered magnetic systems is discussed. This leads to quantum size effects which can be observed by photoemission in overlayers. In magnetic multilayers, spectacular oscillatory behavior of the interlayer exchange coupling results from the electron confinement. The quantum size effects manifest themselves also in the magneto-optical properties of ultrathin films.

PACS numbers: 75.30.Et, 75.70.-i, 75.70.Cn, 78.20.Ls

\section{Introduction}

It is well known that confinement of electron motion leads to a size quantization of the momentum. In solid state physics, this phenomenon has been widely investigated mostly in semiconducting materials; this has led to numerous applications in which the quantum size effects are used to tailor the electrical or optical properties of semiconductor devices [1]. In layered superstructures, the confinement is one-dimensional, with the motion of electrons in the two other directions remaining free; confinement of electrons in semiconductor heterostructures has provided renewed insight into fundamental phenomena such as quantum tunneling [2] and allowed the discovery of fascinating novel effects such as the quantized Hall effect [3]. One can also confine the electrons in two, or even three dimensions, to form systems called, respectively, quantum wires, and quantum boxes.

In contrast to this, the investigation of quantum size effects in metallic magnetic materials can be considered as still being in its infancy. Several reasons can be invoked to explain this situation:

- In semiconductors, the density of carriers is very low, so that the relevant states are usually located very close to the conduction or valence band edge and have wavelengths ranging between 50 and $500 \AA$; thus, the characteristic size for quantum confinement is from a few tens to a few hundreds of angströms. In metals, however, the electron density is very high as compared to semiconductors, and the relevant lengthscale is the Fermi wavelength, which is of the order of the crystalline lattice parameter, i.e., a few angströms. Thus, the observation of quantum size" effects in metals generally requires the preparation of samples with perfectly controlled thicknesses, at the scale of one atomic layer (AL); 
- The growth of metallic superstructures is not yet mastered as well as for semiconductors;

- From the theoretical point of view, the electronic band structure of transition metals is far more complex than the free-electron-like $s p$ bands of semiconductors; this makes the theoretical analysis more complicated.

In spite of the difficulties mentioned above, the study of quantum size effects in layered magnetic systems has attracted considerable attention in the past five years, and encountered a number of significant successes. The aim of this paper is to give an overview of this field.

\section{Quantum size effects in layered magnetic systems}

\subsection{Change of spectral density due to electron confinement in a metallic overlayer}

In metals, the high density of electrons ensures a very efficient screening of the Coulombic potential of defects. Thus, already at a distance of one to two atomic layers away from a surface or an interface, the electrons experience the same potential as in the bulk of the material, and the potential changes significantly only in the immediate neighboring of the surface (resp. interface).

The electrons in a metallic overlayer are confined by the vacuum barrier on one side, and by the substrate barrier on the other side. As we shall see, the electronic states in the overlayer can be described in terms of the band structure of the corresponding bulk material and the reflection coefficients on the vacuum and substrate barriers. Since the in-plane translational invariance is preserved in the layered structure, the in-plane wave vector $k_{\|}$is conserved after the reflection and remains a good quantum number. So, for a given $k_{\|}$, the problem reduces to an effective one-dimensional problem. The perpendicular wave vector of a state with a positive (resp. negative) perpendicular velocity $v_{\perp}$ will be noted by $k_{\perp}^{+}$ (resp. $k_{\perp}^{-}$). The corresponding velocities will be noted as $v_{\perp}^{+}$and $v_{\perp}^{-}$, respectively. The (complex) reflection coefficients on the vacuum and substrate barriers are noted, respectively, by $r_{\mathrm{v}}$ and $r_{\mathrm{s}}$. In general, $r_{\mathrm{v}}$ and $r_{\mathrm{s}}$ are functions of $\varepsilon$ and $k_{\|}$.

The vacuum barrier is perfectly reflecting for electrons having an energy below the vacuum level; thus, the corresponding reflection coefficient has a module $\left|r_{\mathrm{v}}\right|=1$. On the other hand, the module of the reflection coefficient on the substrate, $\left|r_{\mathrm{s}}\right|$ may be smaller than one or equal to one, depending on $\varepsilon$ and $\boldsymbol{k}_{\|}$. If some propagative states are available in the substrate for the corresponding $\varepsilon$ and $k_{\|}$, one generally has $\left|r_{\mathrm{s}}\right|<1$; if there is a local gap in the substrate band structure for the corresponding $\varepsilon$ and $k_{\|}$, then one has a total reflection, i.e., $\left|r_{\mathrm{s}}\right|=1$. Hereafter, the former situation will be referred to as partial confinement, and the latter one as total confinement. In general, $r_{\mathrm{v}}$ and $r_{\mathrm{s}}$ can be expressed as

$$
r_{\mathrm{v}}=\left|r_{\mathrm{v}}\right| \exp \left(\mathrm{i} \Phi_{\mathrm{v}}\right)
$$

and

$$
r_{\mathrm{s}}=\left|r_{\mathrm{s}}\right| \exp \left(\mathrm{i} \Phi_{\mathrm{s}}\right)
$$

where $\Phi_{\mathrm{v}}$ and $\Phi_{\mathrm{s}}$ are the phase shifts associated with the reflection on the vacuum and on the substrate, respectively. 


\subsubsection{Limit case of total confinement}

Let us first examine the situation of total confinement. The phase shift associated with a round trip in the overlayer is given by

$$
\Phi=\Phi_{\mathrm{v}}+\Phi_{\mathrm{s}}+\left(k_{\perp}^{+}-k_{\perp}^{-}\right) D,
$$

where $D=N d$ is the thickness of the metallic overlayer ( $d$ is the thickness of one atomic layer). The allowed states are those for which

$$
\Phi=2 \pi n,
$$

where $n$ is an integer. This is analogous to the Bohr-Sommerfeld quantization of orbits in atoms. Thus the $k_{\|}$-projected density of states (or spectral density), $n\left(k_{\|}, \varepsilon\right)$, consists of a set of sharp peaks. The peak separation is given by

$$
\delta \varepsilon=\frac{2 \pi}{2 N d /\left(\hbar v_{\perp}^{+-}\right)+\mathrm{d} \Phi_{\mathrm{v}} / \mathrm{d} \varepsilon+\mathrm{d} \Phi_{\mathrm{s}} / \mathrm{d} \varepsilon} \approx \frac{\pi \hbar v_{\perp}^{+-}}{N d}
$$

where

$$
\frac{2}{v_{\perp}^{+-}} \equiv \frac{1}{v_{\perp}^{+}}-\frac{1}{v_{\perp}^{-}}
$$

For a given energy, as the thickness of the overlayer is varied, quantized states pass periodically through the energy under consideration, with a period given by*

$$
\Lambda=\frac{2 \pi}{\left|k_{\perp}^{+}-k_{\perp}^{-}\right|} \text {. }
$$

\subsubsection{Limit case of weak confinement}

Let us now discuss the opposite limit case, in which the confinement strength $\left|r_{\mathrm{v}} r_{\mathrm{s}}\right|$ is much smaller than one. Consider an electron of energy $\varepsilon$ and wave function $\psi(r)$ travelling through the overlayer. The electron is reflected on the vacuum and substrate barriers, so that, to the first order in $\left|r_{\mathrm{v}} r_{\mathrm{s}}\right|$, the wave function becomes

$$
\psi^{\prime}(r) \approx \psi(r)\left[1+r_{\mathrm{v}} r_{\mathrm{s}} \mathrm{e}^{\mathrm{i}\left(k_{\perp}^{+}-k_{\perp}^{-}\right) D}+\cdots\right] .
$$

The interferences give rise to a change in the density of states. The relative change $g\left(k_{\|}, \varepsilon\right)$ of spectral density (i.e., normalized to the spectral density in absence of barriers for a thickness $D$ ) can be expressed as

$$
g\left(k_{\|}, \varepsilon\right) \approx \frac{\left|\psi^{\prime}(r)\right|^{2}}{|\psi(r)|^{2}} \approx 1+2 \operatorname{Re}\left[r_{v} r_{\mathrm{s}} \mathrm{e}^{\mathrm{i}\left(k_{\perp}^{+-k_{\perp}}\right) D}\right]+\cdots,
$$

to the lowest order in $\left|r_{\mathrm{s}} r_{\mathrm{v}}\right|$. In the case of weak confinement, the interferences due to reflections on the vacuum and substrate barriers leads to a sine-like modulation of the spectral density $n\left(k_{\|}, \varepsilon\right)$, with the same period

$$
\Lambda=\frac{2 \pi}{\left|k_{\perp}^{+}-k_{\perp}^{-}\right|}
$$

* Since the overlayer thickness varies only by increments of $d$, it is clear that periods $\Lambda$ smaller than $2 d$ are physically meaningless. Thus, in Eq. (7), we have to add $p \pi / d$ to $k_{\perp}^{+}-k_{\perp}^{-}$with $p$ an integer chosen such that $\left|k_{\perp}^{+}-k_{\perp}^{-}+p \pi / d\right| \leq \pi / d$, i.e., such that $A \geq 2 d$; in other words, wave vectors are folded into the first Brillouin zone. Such a procedure will be implicitely meant when writing down an expression like Eq. (7). 
as for the total confinement case, and an amplitude proportional to the confinement strength $\left|r_{\mathrm{s}} r_{\mathrm{v}}\right|$. This is easily understood in terms of interferences. Whenever the phase-shift $\Phi$ corresponding to a round trip in the overlayer (Eq. (3)) is equal to an integer multiple of $2 \pi$, constructive interferences occur, and hence, an increase in the density of states. Conversely, if $\Phi$ is equal to a half-integer multiple of $2 \pi$, the interferences are destructive, leading to a reduction of the density of states.

\subsubsection{General case}

In the general case, the calculation of the relative change of spectral density is more complicated. It can be performed by using the Green's function formalism $[4,5]$. The result is ${ }^{\dagger}$

$$
g\left(k_{\|}, \varepsilon\right)=\operatorname{Re}\left[\frac{1+r_{\mathrm{s}} r_{\mathrm{v}} \mathrm{e}^{\mathrm{i}\left(k_{\perp}^{+}-k_{\perp}^{-}\right) D}}{1-r_{\mathrm{s}} r_{\mathrm{v}} \mathrm{e}^{\mathrm{i}\left(k_{\perp}^{+}-k_{\perp}^{-}\right) D}}\right] .
$$

One can easily check that both limit cases (total confinement and weak confinement) are obtained from this general result. The behavior of $g\left(k_{\|}, \varepsilon\right)$ versus overlayer thickness $D$ is shown in Fig. 1, for the weak, intermediate, and total confinement cases.

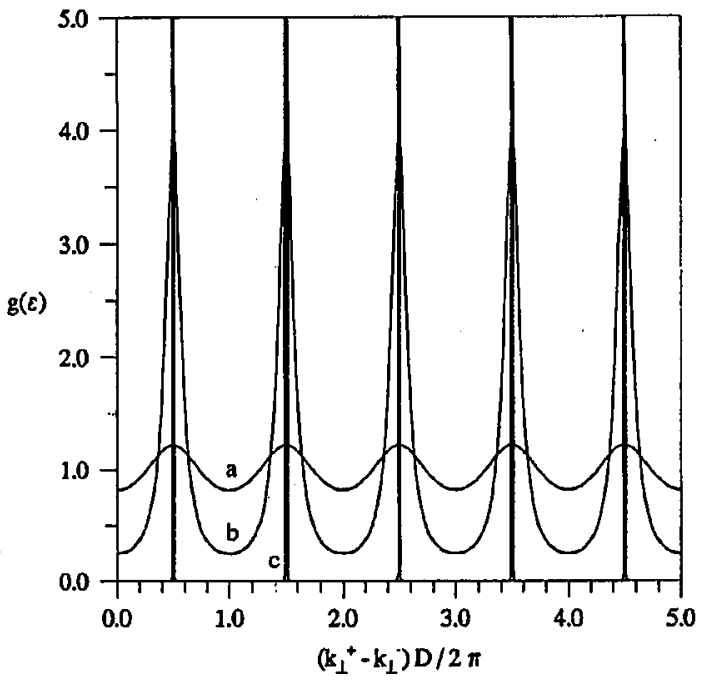

Fig. 1. The variation of the relative change of spectral density due to confinement, as a function of overlayer thickness $D$. The curves labelled $a, b$ and $c$ correspond respectively to the cases of weak confinement $\left(\left|r_{s} r_{v}\right|=0.1\right)$, intermediate confinement $\left(\left|r_{s} r_{v}\right|=0.6\right)$, and total confinement $\left(\left|r_{\mathrm{s}} r_{\mathrm{v}}\right|=1\right)$.

To. summarize, the confinement of electrons in the overlayer leads to an energy and $\boldsymbol{k}_{\|}$dependent modulation of the spectral density versus overlayer thick-

TThis result is valid when the overlayer thickness $D$ is sufficiently large for the energy dependence of the reflection coefficient to be small as compared to the one of the exponential factor in Eq. (11). 
ness. The period depends only on the overlayer bulk band structure, and is given by $\Lambda=2 \pi /\left|k_{\perp}^{+}-k_{\perp}^{-}\right|$. In contrast to this, the phase and amplitude of the modulation depend on the matching of the overlayer wave functions with evanescent waves in the vacuum and with the wave functions in the substrate; the phase is given by $\Phi_{\mathrm{s}}+\Phi_{\mathrm{v}}=\arg \left(r_{\mathrm{s}} r_{\mathrm{v}}\right)$, while the amplitude is determined by the confinement strength $\left|r_{\mathrm{s}} r_{\mathrm{v}}\right|$.

2.2. Photoemission and quantum size effects in magnetic systems

The method which has been most used to investigate quantum size effects is angle-resolved UV photoemission spectroscopy (and to a lesser extent, inverse photoemission). Peaks in the photoemission spectra due to size quantization have been observed in various systems.

\subsubsection{Ferromagnetic overlayer}

In a ferromagnetic transition metal, the exchange interaction leads to a splitting between the $3 d$ bands of opposite spin directions. Thus, the effective potential experienced by the electrons is spin-dependent. In the bulk-like region of the ferromagnetic overlayer, the majority and minority spin electrons of energy $\varepsilon$ and in-plane wave vector $k_{\|}$propagate with a perpendicular wave vector $k_{\perp}^{ \pm \uparrow}$ and $k_{\perp}^{ \pm \downarrow}$, respectively. Furthermore, the spin-dependence of the potential in the overlayer implies that the reflection coefficients on the vacuum and substrate barriers are also spin-dependent.

The effect of confinement of electrons in the overlayer due to reflections on the vacuum and substrate barriers is given by the same expression as for non-magnetic systems (Eq. (11)), by replacing respectively $g\left(k_{\|}, \varepsilon\right), r_{\mathrm{v}}, r_{\mathrm{s}}$, and $k_{\perp}^{ \pm}$, by $g^{\sigma}\left(k_{\|}, \varepsilon\right), r_{\mathrm{v}}^{\sigma}, r_{\mathrm{s}}^{\sigma}$, and $k_{\perp}^{ \pm \sigma}$, where $\sigma=(\uparrow, \downarrow)$ is the spin index.

As for the non-magnetic case, the confinement leads to a periodic modulation of the spectral density versus overlayer thickness; however, in the magnetic case, the period, the amplitude, and the phase of the modulation depends on the spin of the electron.

Only a few number of experimental investigations of quantum size effects in ferromagnetic overlayers have been published so far. A notable exception is the work of Clemens et al. [6], who performed spin-polarized photoemission investigations of quantum size effects in Co overlayers on $\mathrm{Cu}(001)$. They observed spin-polarized quantum size effects, from which they determined the spin-resolved band structure of fcc Co along the $\Gamma-\Delta-X$ high-symmetry line.

\subsubsection{Paramagnetic overlayer on a ferromagnetic substrate}

The case of a paramagnetic overlayer on a ferromagnetic substrate has been investigated much more. In the bulk-like region of the overlayer, the propagation of the electrons is governed by the $k_{\perp}^{ \pm}$wave vectors which are spin-independent. The reflection coefficient on the vacuum potential barrier, $r_{\mathrm{v}}$, is also spin-independent. However, the ferromagnetic substrate constitutes a spin-dependent potential barrier; thus the substrate reflection coefficients for electrons with a spin parallel to the majority and minority spin directions in the substrate, respectively $r_{\mathrm{s}}^{\uparrow}$ and $r_{\mathrm{s}}^{\perp}$, will be different. It is convenient to define, respectively, the spin average as

$$
\bar{r}_{\mathrm{s}} \equiv \frac{r_{\mathrm{s}}{ }^{\uparrow}+r_{\mathrm{s}}^{\downarrow}}{2}
$$


and the spin asymmetry as

$$
\Delta r_{\mathrm{s}} \equiv \frac{r_{\mathrm{s}}^{\uparrow}-r_{\mathrm{s}}^{\downarrow}}{2}
$$

In this case, the electron confinement in the overlayer gives rise to a spin-dependent modulation of the spectral density versus overlayer thickness; the period of the modulation is the same for both spins, whereas the amplitude and phase are expected to be spin-dependent.

The quantum size effects in paramagnetic overlayers on a ferromagnetic substrate have been investigated by several groups [7-15]. The systems studied most are $\mathrm{Cu}$ overlayers on a $\mathrm{Co}(001)$ substrate and $\mathrm{Ag}$, overlayers on a $\mathrm{Fe}(001)$ substrate.

Ortega and Himpsel [8] have proposed that the quantum size effect observed near and below the Fermi level are spin-polarized and primarily due to minority-spin electrons. However, only a spin-polarized experiment could establish this unambiguously. The suggestion of Ortega and Himpsel has been confirmed independently by Garrison et al. [10] and by Carbone et al. [11] who performed spin-polarized photoemission studies of $\mathrm{Cu}$ films on $\mathrm{Co}(001)$.

\section{Interlayer exchange coupling}

\subsection{General considerations}

In metallic systems, exchange interactions are propagated by itinerant electrons and can thus be transmitted over rather long distances. It follows that exchange interactions can couple ferromagnetic layers through a non-magnetic metallic spacer layer. Although the possibility of exchange coupling across a non-magnetic spacer has been considered for a long time, it was only clearly identified in 1986 in rare-earth multilayers by Salamon et al. [16] and by Majkrzak et al. [17], and in $\mathrm{Fe} / \mathrm{Cr} / \mathrm{Fe}$ films by Grünberg et al. [18]. In 1990, Parkin et al. [19] observed periodic oscillations versus spacer thickness of the interlayer exchange coupling (IEC) in $\mathrm{Co} / \mathrm{Ru}$ and $\mathrm{Co} / \mathrm{Cr}$ multilayers. This discovery gave a decisive impetus to this field and several hundreds of papers on this subject have been published since then. In a systematic study, Parkin showed that oscillatory exchange coupling occurs with almost all transition or noble metals as a spacer material [20].

In most cases, the exchange coupling energy per unit area between two ferromagnetic films $F_{A}$ and $F_{B}$ separated by a non-magnetic spacer can be expressed as

$$
E_{A, B}=J \cos \theta \text {, }
$$

where $\theta$ is the angle between the magnetizations in $F_{A}$ and $F_{B}$. With the convention used in Eq. (14), the positive (resp. negative) sign of $J$ corresponds to an antiferromagnetic (resp. ferromagnetic) coupling ${ }^{\ddagger}$. The typical order of magnitude of IEC ranges between 0.1 and $1 \mathrm{erg} \mathrm{cm}^{-2}$ for a spacer thickness of $5 \mathrm{AL}$ ( $1 \mathrm{erg} \mathrm{cm}^{-2}=1 \mathrm{~mJ} \mathrm{~m}^{-2}$ ).

Although in most cases, the coupling is of the form (14), which leads to either ferro- or antiferromagnetic coupling, Rührig et al. [21] found that for spacer thicknesses around a change of sign of $J$, the interlayer coupling in $\mathrm{Fe} / \mathrm{Cr} / \mathrm{Fe}$ films

$\$$ Note that different conventions for the sign and/or dimension of the coupling constant $J$ are frequently found in the literature. 
leads to a $90^{\circ}$-angle between the magnetizations of the Fe layers. This effect (called biquadratic coupling) can be accounted for by adding to Eq. (14) a term $B \cos ^{2} \theta$, with $B>0$. The same effect has been also observed in other systems.

A great variety of experimental methods have been used to investigate IEC:

- The most widely used method consists in measuring magnetization [20,22], magneto-optical Kerr effect (MOKE) [23, 24] or magnetoresistance [19] loops. With this method, however, only antiferromagnetic coupling can be measured in general. In order to be able to measure also ferromagnetic coupling, one has to use a special sample design [22,24]. A particular advantage of MOKE is that it is easily implemented in vacuo and that it can be used to probe locally the coupling in samples with a wedge or staircase spacer layer (produced by moving a shutter during evaporation). This procedure allows a very fine investigation of the spacer thickness dependence of the coupling, and avoids problems due to irreproducibily in sample preparation conditions;

- RF techniques such as ferromagnetic resonance (FMR) [25] and Brillouin light scattering (BLS) [25-27] have been also used;

- Neutron scattering [28-29] has been used to evidence antiferromagnetic coupling in multilayers;

- A spectacular technique to evidence IEC oscillations consists in imaging the magnetic domains in a system with a wedge-shaped spacer by means of a scanning electron microscope with a spin-polarization analysis (SEMPA) [30-32].

From the theoretical point of view, an intense activity has been devoted to the problem of IEC. The most direct way to study theoretically the IEC is to compute the total energy difference between the ferromagnetic and antiferromagnetic configurations [33-37]. Although this is in principle straightforward, this approach turns out to be quite difficult. This is due in particular to the fact that IEC energies are of the order of $10^{-4}$ to $10^{-3} \mathrm{eV}$ per unit cell, i.e., considerably smaller than the total energy of the system. Indeed, early attempts of computing IEC from first-principles yielded coupling strengths much larger than the experimental ones. However, recent calculations are now in much better agreement with experimental results.

In parallel with first-principle calculations, various models have been proposed to study the IEC:

- the Ruderman-Kittel-Kasuya-Yoshida (RKKY) model in which the magnetic layers are described as arrays of localized spins interacting with conduction electrons by a contact exchange potential [38-42];

- the free-electron model [43-46];

- the hole confinement model, which is essentially a tight-binding model with spin-dependent potential steps [47-48];

- the Anderson (sd-mixing) model [49-50]. 
Recently, it has been shown that all the above models can be unified into a more general approach in which the IEC is interpreted as a quantum size effect and described in terms of (spin-dependent) reflection coefficients of electrons at the interfaces between the non-magnetic spacer and the ferromagnetic layers $[4,5,51]$. This approach will be presented in the following section.

Note that, in the following, only bilinear IEC such as expressed by Eq. (14) will be considered. Although the quantum size effect does give rise to biquadratic and higher-order terms, their contribution is too small to explain the biquadratic coupling observed experimentally [21]. Several non-intrinsic mechanisms have been proposed to explain the biquadratic coupling [52].

\subsection{Interpretation of interlayer exchange coupling as a quantum size effect}

In this section, the interpretation of IEC as a quantum size effect, and the description in terms of spin-dependent reflection at the spacer-ferromagnet interfaces will be presented. For a comprehensive discussion, however, the reader is referred to the original publications [4-5].

Let us consider a paramagnetic spacer layer sandwiched between two ferromagnetic films $F_{A}$ and $F_{B}$. The latter constitute potential barriers for the electrons travelling through the spacer layer, so that partial or complete confinement of electrons in the spacer takes place. Thus, as discussed above, the density of states of the system is modified by the quantum size effect. This in turn leads to a change in the total energy of the system. The reflection coefficients at the interfaces between the paramagnetic spacer and the ferromagnetic layers are spin dependent, so that the quantum size effect (and, hence, the total energy of the system) depends on the relative alignment of the magnetizations in $F_{A}$ and $F_{B}$. This is the physical origin of the IEC. Since the quantum size effect results from quantum interferences in the spacer, one can easily understand that, upon variation of spacer thickness, situations of constructive and destructive interferences will alternate periodically, resulting into an oscillatory behavior of the IEC.

Let $\Delta n(\varepsilon)$ be the total change of density of states (per unit area) of the system, resulting from the quantum size effect in the spacer layer. The associated change in the total energy (per unit area) of the system can be expressed as

$$
\Delta E=\int_{-\infty}^{\varepsilon_{\mathrm{F}}}\left(\varepsilon-\varepsilon_{\mathrm{F}}\right) \Delta n(\varepsilon) \mathrm{d} \varepsilon
$$

In the above equation, the second term ensures conservation of the total number of electrons in the system; this prescription corresponds to working in the grand-canonical ensemble, i.e., with a fixed chemical potential $\varepsilon_{F}$. The IEC constant $J$ can be obtained from $E_{\mathrm{F}}-E_{\mathrm{AF}}=2 J$, where the subscripts $\mathrm{F}$ and $\mathrm{AF}$ correspond respectively to the ferromagnetic and antiferromagnetic configurations of the magnetizations in $F_{A}$ and $F_{B}$. Thus

$$
E_{\mathrm{F}}-E_{\mathrm{AF}}=\int_{-\infty}^{\varepsilon_{\mathrm{F}}}\left(\varepsilon-\varepsilon_{\mathrm{F}}\right)\left[\Delta n_{\mathrm{F}}(\varepsilon)-\Delta n_{\mathrm{AF}}(\varepsilon)\right] d \varepsilon
$$

For simplicity, the approximation corresponding to the weak confinement case is made. An exact treatment of the general case is given in Refs. [4-5]. For the weak 
confinement case, by using Eq. (9) and performing an integration by parts on the energy, one obtains

$$
\begin{gathered}
E_{\mathrm{F}}-E_{\mathrm{AF}} \approx-\frac{1}{4 \pi^{3}} \operatorname{Im} \int \mathrm{d}^{2} k_{\|} \int_{-\infty}^{\varepsilon_{\mathrm{F}}}\left[\left(r_{A}^{\uparrow} r_{B}^{\uparrow}+r_{A}^{\downarrow} r_{B}^{\downarrow}\right)\right. \\
\left.-\left(r_{A}^{\uparrow} r_{B}^{\downarrow}+r_{A}^{\downarrow} r_{B}^{\uparrow}\right)\right] e^{i\left(k_{\perp}^{+}-k_{\perp}^{-}\right) D} d \varepsilon .
\end{gathered}
$$

In the above equation, the first term correspond to the ferromagnetic configuration and the second term to the antiferromagnetic one; the integration on $k_{\|}$is performed over the first two-dimensional Brillouin zone. The indices $A$ and $B$ label the reflection coefficients on the ferromagnetic layers $F_{A}$ and $F_{B}$, respectively, corresponding to an electron travelling through the spacer with an in-plane wave vector $k_{\|}$and an energy $\varepsilon$. Finally, this yields

$$
E_{\mathrm{F}}-E_{\mathrm{AF}} \approx-\frac{1}{\pi^{3}} \operatorname{Im} \int \mathrm{d}^{2} k_{\|} \int_{-\infty}^{\varepsilon_{F}} \Delta r_{A} \Delta r_{B} \mathrm{e}^{\mathrm{i}\left(k_{\perp}^{+}-k_{\perp}^{-}\right) D} \mathrm{~d} \varepsilon
$$

The above expression for the IEC has a rather transparent physical interpretation. First, as the integrations on $k_{\|}$over the first two-dimensional Brillouin zone and on the energy up to the Fermi level show, the IEC is a sum of contributions from all occupied electronic states. The contribution of a given electronic state, of energy $\varepsilon$ and in-plane wave vector $\boldsymbol{k}_{\|}$, consists of the product of three factors: the two factors $\Delta r_{A}$ and $\Delta r_{B}$ express the spin-asymmetry of the confinement due to $F_{A}$ and $F_{B}$, respectively, while the exponential factor $\mathrm{e}^{\mathrm{i}\left(k_{\perp}^{+}-k_{\perp}^{-}\right) D}$ describes the propagation through the spacer and is responsible for the interference (or quantum size) effect. Thus, this approach establishes an explicit and direct link between oscillatory IEC and quantum size effects such as observed in photoemission.

It should be emphasized that the dependence of the coupling on the nature and thickness of the ferromagnetic layers is entirely described via the factors $\Delta r_{A}$ and $\Delta r_{B}{ }^{\S}$. On the other hand, the dependence of the IEC on the spacer layer thickness is determined only by the (bulk) band structure of the spacer material, via the wave vectors $k_{\perp}^{+}$and $k_{\perp}^{-}$(which are, of course, functions of $\varepsilon$ and $k_{\|}$).

In the limit of the large spacer thickness $D$, the exponential factor oscillates rapidly with $\varepsilon$ and $k_{\|}$, which leads to some cancellation of the contributions to the IEC due to the different electronic states. However, because the integration over energy is abruptly stopped at $\varepsilon_{\mathbf{F}}$, states located at the Fermi level give predominant contributions. A detailed analysis [4-5,41] shows that in fine, the only remaining terms in the limit of the large spacer thickness $D$ arise from the neighborhood of states having in-plane wave vectors $k_{i l}^{\alpha}$ such that the spanning vector of the Fermi surface $q_{\perp \mathrm{F}}=k_{\perp \mathrm{F}}^{+}-k_{\perp \mathrm{F}}^{-}$is stationary with respect to $k_{\|\|}$for $k_{\|}=k_{\|}^{\alpha}$, and the corresponding contribution oscillates with a wave vector equal to $q_{\perp \mathrm{F}}^{\alpha}$ ?. This

\footnotetext{
${ }^{\S}$ Actually, the reflection coefficients on $F_{A}$ and $F_{B}$, respectively, incorporate the effect of interferences due to the whole half-spaces on each side of the spacer layer, so that they also contain the possible influence of layers (substrate, protective cap layer, or vacuum) located "behind" $F_{A}$ and $F_{B}$.

IThere may be several such stationary spanning vectors and, hence, several oscillatory components; they are labelled by the index $\alpha$.
} 
selection rule was first derived in the context of the RKKY model [41]. Examples will be given in the next section.

\subsection{Theoretical predictions and comparison with experiment}

\subsubsection{Oscillations versus spacer layer thickness}

The easiest comparison between the above theory and the experimental observations concerns the periods of oscillation of the IEC with respect to a spacer layer thickness. As already mentioned, the theory predicts that the oscillation periods depend only on the bulk band structure of the spacer material. Thus noble metals constitute very good candidates for an experimental test of the oscillation periods predicted by the theory; there are several reasons for this choice:

- Fermi surfaces of noble metals are known very accurately from de Haas-van Alphen and cyclotron resonance experiments [53];

- Since only the $s p$ band intersects the Fermi level, the Fermi surface is rather simple, and does not depart very much from a free-electron Fermi sphere;

- Samples of a very good quality with noble metals as a spacer layer could be prepared.

Figure 2 shows a cross-section of the Fermi surface of $\mathrm{Cu}$, indicating the stationary spanning vectors for the (001), (111), and (110) crystalline orientations [41]; the Fermi surfaces of $\mathrm{Ag}$ and $\mathrm{Au}$ are qualitatively similar. For the (111) orientation, a single (long) period is predicted; for the (001) orientation, both a long period and a short period are predicted; for the (110) orientation, four different periods are predicted (only one stationary spanning vector is seen in Fig. 2, the three others being located in other cross-sections of the Fermi surface). These

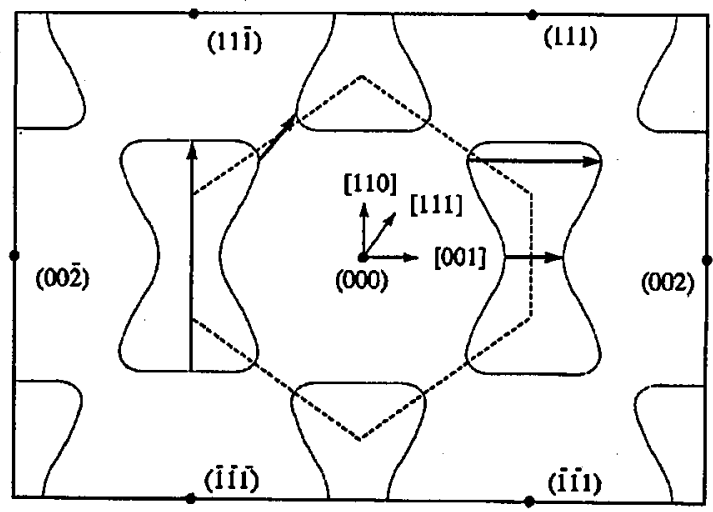

Fig. 2. Cross-section of the Fermi surface of $\mathrm{Cu}$, parallel to a (1ī0) section. The solid points give the vectors of the reciprocal lattice. The (bulk) first Brillouin zone is indicated by the dashed lines. The horizontal, oblique, and vertical bold arrows, respectively, are the stationary spanning vectors determining the periods of IEC oscillations versus spacer thickness, for the (001), (111) and (110) orientations (from Ref. [41]). 
theoretical predictions have been confirmed successfully by numerous experimental observations. In particular, the coexistence of a long and a short period for the (001) orientation has been confirmed for $\mathrm{Cu}[54], \mathrm{Ag}$ [55], and $\mathrm{Au}[31,56]$.

In a further attempt to test the theoretical predictions for the periods of oscillatory coupling, several groups [57-59] have undertaken to modify in a controlled manner the size of the Fermi surface (and hence, the period of the coupling) by alloying the spacer noble metal $(\mathrm{Cu})$ with a metal of lower valence $(\mathrm{Ni})$; in both cases, the change in oscillation period due to alloying has been found in good agreement with the expected change in the Fermi surface.

While a test of the theory with respect to oscillation periods is rather easy because it only requires an inspection of the Fermi surface, a quantitative discussion of the magnitude and phase of the IEC is a more difficult task, because it requires the calculation of reflection coefficients at the spacer/ferromagnet interface. Such calculations, based upon the theory outlined above, have been performed by Lee and Chang [60] and by Stiles [61] for various systems. Their results are in rather good agreement, both with total energy ab initio calculations and with experiments.

In contrast to the success obtained with noble metal spacer layers, the situation for transition metal spacer layers is much less satisfactory. Stiles [51] has performed systematic calculations of the possible oscillation periods for transition metal spacers. However, due to the fact that the $d$-bands intersect the Fermi level, the Fermi surfaces are very complicated and consist of several sheets. As a consequence, so many possible periods are predicted that a reliable comparison with experimental data is problematic.

\subsubsection{Variation versus ferromagnet and overlayer thicknesses}

As already mentioned, the influence of the IEC on the ferromagnetic layer thickness is contained in the reflection coefficients $\Delta r_{A}$ and $\Delta r_{B}$. If the ferromagnetic layers are of a finite thickness, reflections usually may take place at the two interfaces bounding the ferromagnetic layers, giving rise to interferences [62], and hence, to oscillations of the IEC versus ferromagnetic layers thickness. A more detailled discussion of this effect is given in Refs. [5,62]. This behavior was found previously from calculations based upon a free-electron model [43], and confirmed by first-principle calculations for the $\mathrm{Co} / \mathrm{Cu}(001)$ system [36]. On the experimental point of view, it was confirmed by Bloemen et al. [63] in $\mathrm{Co} / \mathrm{Cu} / \mathrm{Co}(001)$ and by Okuno and Inomata [64] in $\mathrm{Fe} / \mathrm{Cr} / \mathrm{Fe}(001)$. The amplitude of the oscillations of the IEC versus ferromagnetic layers thickness is generally much smaller than the oscillations versus spacer thickness, and does not give rise to changes of sign of the IEC.

A more (a priori) surprising behavior is the dependence of the IEC on the thickness of the protective overlayer. From a naïve point of view, one might think that layers external to the basic ferromagnet/spacer/ferromagnet sandwich should not influence the interaction between the two ferromagnetic layers. This view is incorrect, in particular when the system is covered by an ultrathin protective overlayer. In this case, the electrons are able to reach the vacuum barrier, which is a perfectly reflecting one, so that strong confinement and interference effects take 
place in the overlayer, which lead to a weak but sizeable oscillatory variation of the IEC as a function of the overlayer thickness. This effect, which follows directly from the quantum interference (or quantum size effect) mechanism, has been proposed and experimentally confirmed independently by de Vries et al. [65] for the $\mathrm{Co} / \mathrm{Cu} / \mathrm{Co}(001)$ system with a $\mathrm{Cu}(001)$ overlayer, by Okuno and Inomata [66] for the $\mathrm{Fe} / \mathrm{Au} / \mathrm{Fe}(001)$ system with a $\mathrm{Au}(001)$ overlayer, and by Bounouh et al. [67] for the $\mathrm{Co} / \mathrm{Au} / \mathrm{Co}(0001)$ with a $\mathrm{Au}(111)$ overlayer. In both cases, the observed period(s) for the oscillations versus overlayer thickness were found to be in good agreement with the theoretically predicted ones.

\section{Magneto-optical effects in ultrathin films}

The optical properties of ferromagnetic materials depend on the relative orientations of the magnetization and of the light polarization. These effects are known as magneto-optical effects. Here, only the simplest of these effects (the polar magneto-optical Kerr effect) will be discussed. When a ferromagnetic (or ferrimagnetic) sample, with its magnetization aligned perpendicular to its surface, is illuminated at (quasi) normal incidence with linearly polarized light, one usually observes that the polarization of the reflected light has acquired an ellipticity $\varepsilon_{\mathrm{K}}$ (called the Kerr ellipticity) and that the large axis of the polarization ellipse is at a certain angle $\theta_{\mathrm{K}}$ (called the Kerr rotation) with respect to the polarization direction of the incident light. If the magnetization is reversed, then the Kerr rotation and ellipticity are also reversed. When the sample is transparent, a similar effect can be also observed on the transmitted light, and this is known as the magneto-optical Faraday effect. Another effect, which is closely related to the polar Kerr effect and to the Faraday effect, is the optical magnetic circular dichroism, i.e., the difference in absorption between left and right circularly polarized light.

\subsection{Kerr effect and conductivity tensor}

On the macroscopic level, the polar magneto-optical Kerr effect is described in terms of the optical conductivity tensor $\sigma(\omega)$ of the magnetic material. The magneto-optical Kerr effect arises from the presence of a non-zero off-diagonal matrix element, $\sigma_{x y}(\omega)$; this may be understood rather easily from the following argument. Let us consider an incident light wave with the electric field linearly polarized along the $x$ axis. The current induced in the ferromagnetic material by the electric field of the light comprises two components: one parallel to the $x$ axis and proportional to the diagonal matrix element $\sigma_{x x}(\omega)$, and the other parallel to the $y$ axis and proportional to the off-diagonal matrix element $\sigma_{x y}(\omega)$. The reflected light is the light radiated by the induced current; thus, its polarization plane is rotated by a small angle, of the order of $\left|\sigma_{x y}(\omega) / \sigma_{x x}(\omega)\right|$, with respect to that of the incident wave.

The Kerr rotation angle $\theta_{\mathrm{K}}$ and the Kerr ellipticity $\varepsilon_{\mathrm{K}}$ may be combined to form the complex Kerr angle

$$
\phi_{\mathrm{K}} \equiv \theta_{\mathrm{K}}+\mathrm{i} \varepsilon_{\mathrm{K}} \text {. }
$$

For a film of thickness $D$ on a substrate, the Kerr rotation angle is [68]

$$
\phi_{\mathrm{K}}=\frac{\mathrm{i} \sigma_{x y}}{\sigma^{\mathrm{s}}{ }_{x x}} \frac{4 \pi D}{\lambda},
$$


where $\sigma_{x x}^{\mathrm{s}}$ is the optical conductivity of the substrate, and $\lambda=2 \pi c / \omega$ the wavelength of the light in vacuum. This expression is valid when $D \ll \lambda$.

\subsection{Microscopic mechanism of magneto-optical Kerr effect in bulk ferromagnets}

Before addressing the question of the magneto-optical effects in ultrathin films, it is useful to present the mechanism of the Kerr effect for the case of bulk ferromagnets.

The expression of the conductivity tensor in terms of the microscopic electronic structure may be obtained from the Fermi golden rule $[69,70]$, or by using the Kubo-Greenwood formalism [71]. The dissipative component of $\sigma_{x y}(\omega)$ (for $\omega>0)$ is then given as [69]

$$
\begin{aligned}
\sigma_{x y}^{\prime \prime}(\omega)=\frac{\pi e^{2}}{4 \hbar \omega m^{2} \Omega} \sum_{i, f} f\left(\varepsilon_{i}\right)\left[1-f\left(\varepsilon_{f}\right)\right] \\
\times\left[\left|\left\langle i\left|p_{-}\right| f\right\rangle\right|^{2}-\left|\left\langle i\left|p_{+}\right| f\right\rangle\right|^{2}\right] \delta\left(\omega_{f i}-\omega\right),
\end{aligned}
$$

where $p_{ \pm} \equiv p_{x} \pm \mathrm{i} p_{y}, f(\varepsilon)$ is the Fermi-Dirac function, $\Omega$ the total volume, and $\hbar \omega_{f i} \equiv \varepsilon_{f}-\varepsilon_{i}$.

The above expression is interpreted straightforwardly in terms of the absorption of a photon by an electron making a transition between an occupied initial state $|i\rangle$ and an unoccupied final state $|f\rangle$; the factor $\delta\left(\omega_{f i}-\omega\right)$ expresses the condition of energy conservation. The matrix elements $\left\langle i\left|p_{-}\right| f\right\rangle$ and $\left\langle i\left|p_{+}\right| f\right\rangle$ correspond to dipolar electric transitions, for right and left circularly polarized light, respectively. Clearly, $\sigma_{x y}^{\prime \prime}(\omega)$ is proportional to the difference of absorption probability for right and left circularly polarized light. This illustrates the deep connection between the Kerr effect and the circular dichroism. The corresponding dispersive component, $\sigma_{x y}^{\prime}(\omega)$ is obtained by using the Kramers-Kronig relation [72].

In a bulk material, due to a three-dimensional translational invariance, the matrix elements vanish unless the initial state $|i\rangle$ and final state $|f\rangle$ of the optical transition have the same wave vector $k$ (as usually, the much smaller wave vector $\boldsymbol{K}$ of the photon is neglected), i.e., only vertical optical transitions are allowed. fied, i.e.,

In addition, the selection rules for electric dipolar transitions must be satis-

$$
\begin{aligned}
& \Delta l= \pm 1 \\
& \Delta m_{l}= \pm 1 .
\end{aligned}
$$

The first selection rule implies that only transitions between $s$ and $p$ levels, or between $p$ and $d$ levels (for transition metals) are allowed. For the second selection rule, the transitions with $\Delta m_{l}=+1$ and $\Delta m_{l}=-1$ correspond to left and right circularly polarized light, respectively. As an example, let us consider a transition between a doubly degenerate $d_{x z, y z}$ level $\left(l=2, m_{l}= \pm 1\right)$ and a $p_{z}$ level $\left(l=1, m_{l}=0\right)$. The majority and minority spin $d$ levels, in the ferromagnet, are separated by the exchange spitting $\Delta_{\text {ex }}$. Due to spin-orbit coupling, the orbital degeneracy of the $d_{x z, y z}$ levels is lifted, and the latter are splitted into $d_{(x+\mathrm{i} y) z}$ (having $m_{l}=+1$ ) and $d_{(x-\mathrm{i} y) z}$ (having $m_{l}=-1$ ) levels. For spin up (i.e., majority spin), the level with $m_{l}=+1$ has a higher energy, whereas for spin down (i.e., 


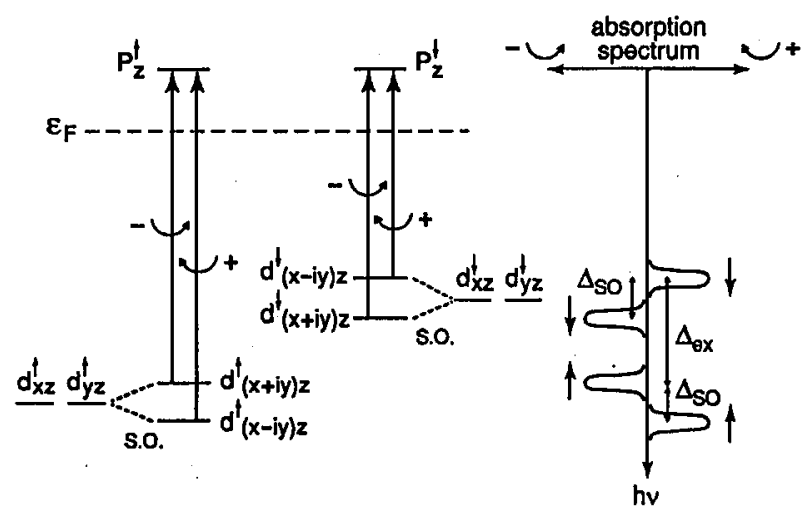

Fig. 3. Sketch of the energy levels in a bulk ferromagnet, showing the electric dipolar optical transitions for left and right circularly polarized light. The corresponding absorption spectra versus photon energy $h \nu$ are shown on the right; (from Ref. [76]).

minority spin), the converse holds. This is sketched in Fig. 3. From this picture, it is clear that, in a bulk ferromagnet, the Kerr effect arises from the simultaneous occurrence of exchange splitting and spin-orbit coupling.

\subsection{Magneto-optical properties of ultrathin films: the effect of electron confinement}

Recently, it has been discovered by Suzuki et al. [68-73] and Geerts et al. [74] that the magneto-optical effects in ultrathin Fe films are modified with respect to those of bulk $\mathrm{Fe}$ (or thick Fe films), due to their modified electronic structure. As will be discussed below, these observations can be interpreted as a quantum size effect due to electron confinement in the Fe film.

A very interesting observation has been done by Mégy et al. [75], who reported an oscillatory behavior of the Kerr rotation versus Au overlayer thickness in the $\mathrm{Au} / \mathrm{Co} / \mathrm{Au}(111)$ system. The latter effect is attributed to Kerr effect in the Au overlayer, due to a spin-dependent quantum size effect $[75,76]$.

As was discussed above, the effect of electron confinement in an ultrathin film is to modulate the spectral density corresponding to a given electronic state by a periodic function of the thickness. The matrix elements for the ultrathin film case $\left\langle i\left|p_{ \pm}\right| f\right\rangle$ are thus modified with respect to those corresponding to the bulk case, $\left\langle i\left|p_{ \pm}\right| f\right\rangle_{0}$. Clearly, this change must be of the form

$$
\left|\left\langle i\left|p_{ \pm}\right| f\right\rangle\right|^{2} \approx\left|\left\langle i\left|p_{ \pm}\right| f\right\rangle_{0}\right|^{2} g(i) g(f),
$$

where $g(i)$ and $g(f)$ are the relative changes of the spectral weight due to the confinement for the initial and final states, respectively. One can then show that $\sigma_{x}^{\prime \prime} y(\omega)$ comprises, in addition to the bulk contribution, an oscillatory term due to quantum confinement in the initial and/or final states of the transition. As in the problem of IEC, oscillatory contributions with different wave vectors tend to cancel each other, except for some specific points in the Brillouin zone [76]. However, the quantitative discussion of the periods of oscillations of the Kerr effect is much 
more difficult than for the IEC, and a quantitative comparison between theory and experiment has not been obtained yet.

\section{Conclusion}

As discussed throughout this chapter, the quantum size effects arising from the confinement of electrons in magnetic ultrathin films manifest themselves in a number of physical properties.

The method of choice for a quantitative investigation of quantum confinement in metals in undoubtedly photoemission; this technique bears unique features that allow selectivity in wave vector, energy, and possibly, spin. Photoemission experiments in overlayers can be analyzed in terms of simple, physically appealing, concepts such as reflection coefficients and bulk band structures.

The oscillatory interlayer exchange coupling has been widely investigated experimentally. From the theoretical point of view, it has been successfully interpreted in terms of quantum size effects, and numerous theoretical predictions have been confirmed experimentally.

The magneto-optical effects in ultrathin films are also sensitive to the quantum size effects. In spite of some recent efforts to explain the observed phenomena, further investigations (both experimental and theoretical) are required in order to confirm the explanations that have been proposed.

\section{References}

[1] C. Weisbuch, B. Vinter, Quantized Semiconductor Structures: Physics and Applications, Academic Press, Boston 1991.

[2] L. Esaki, Rev. Mod. Phys. 46, 237 (1974).

[3] K. von Klitzing, Rev. Mod. Phys. 58, 519 (1986).

[4] P. Bruno, J. Magn. Magn. Mater. 121, 248 (1993).

[5] P. Bruno, Phys. Rev. B 52, 411 (1995).

[6] W. Clemens, T. Kachel, E. Vescovo, S. Blügel, C. Carbone, W. Eberhardt, Solid State Commun. 81, 739 (1992).

[7] N.B. Brookes, Y. Chang, P.D. Johnson, Phys. Rev. Lett. 67, 354 (1991).

[8] J.E. Ortega, F.J. Himpsel, Phys. Rev. Lett. 69, 844 (1992).

[9] J.E. Ortega, F.J. Himpsel, G.J. Mankey, R.F. Willis, Phys. Rev. B 47, 1540 (1993); J. Appl. Phys. 73, 5771 (1993).

[10] K. Garrison, Y. Chang, P.D. Johnson, Phys. Rev. Lett. 71, 2801 (1993).

[11] C. Carbone, E. Vescovo, O. Rader, W. Gudat, W. Eberhardt, Phys. Rev. Lett. 71, 2805 (1993).

[12] N.V. Smith, N.B. Brookes, Y. Chang, P.D. Johnson, Phys. Rev. B 49, 332 (1994).

[13] P.D. Johnson, K. Garrison, Q. Dong, N.V. Smith, D. Li, J. Mattson, J. Pearson, S.D. Bader, Phys. Rev. B 50, 8954 (1994).

[14] D. Li, J. Pearson, J.E. Mattson, S.D. Bader, P.D. Johnson, Phys. Rev. B 51, 7195 (1995).

[15] F.J. Himpsel, O. Rader, Appl. Phys. Lett. 67, 1151 (1995).

[16] M.B. Salamon, S. Sinha, J.J. Rhyne, J.E. Cunningham, R.W. Erwin, J. Borchers, C.P. Flynn, Phys. Rev. Lett. 56, 259 (1986). 
[17] C.F. Majkrzak, J.W. Cable, J. Kwo, M. Hong, D.B. McWan, Y. Yafet, J.W. Waszczak, C. Vettier, Phys. Rev. Lett. 56, 2700 (1986).

[18] P. Grünberg, F. Schreiber, Y. Pang, M.B. Brodsky, H. Sower, Phys. Rev. Lett. 57, 2442 (1986).

[19] S.S.P. Parkin, N. More, K.P. Roche, Phys. Rev. Lett. 64, 2304 (1990).

[20] S.S.P. Parkin, Phys. Rev. Lett. 67, 3598 (1991).

[21] M. Rührig, M. Schäfer, A. Hubert, R. Mosler, J.A. Wolf, S. Demokritov, P. Grünberg, Phys. Status Solidi A 125, 635 (1991).

[22] S.S.P. Parkin, D. Mauri, Phys. Rev. B 44, 7131 (1991).

[23] S.T. Purcell, W. Folkerts, M.T. Johnson, N.W.E. McGee, K. Jager, J. aan de Stegge, W.B. Zeper, W. Hoving, P. Grünberg, Phys. Rev. Lelt. 67, 903 (1991).

[24] V. Grolier, D. Renard, B. Bartenlian, P. Beauvillain, C. Chappert, C. Dupas, J. Ferré, M. Galtier, É. Kolb, M. Mulloy, J.-P. Renard, P. Veillet, Phys. Rev. Lett. 71, 3023 (1993).

[25] Z. Celinski, B. Heinrich, J.F. Cochran, W.B. Muir, A.S. Arrott, J. Kirschner, Phys. Rev. Lett. 65, 1156 (1990).

[26] S. Demokritov, J.A. Wolf, P. Grünberg, Europhys. Lett. 15, 881 (1991).

[27] Y. Roussigné, F. Ganot, C. Dugautier, P. Moch, D. Renard, Phys. Rev. B 52, 350 (1995).

[28] B. Rodmacq, P. Mangin, C. Vettier, Europhys. Lett. 15, 503 (1991).

[29] A. Schreyer, K. Bröhl, J.F. Ankner, C.F. Majkrzak, Th. Zeidler, P. Bödeker, N. Metoki, H. Zabel, Phys. Rev. B 47, 15334 (1993).

[30] J. Unguris, R.J. Celotta, D.T. Pierce, Phys. Rev. Lett. 67, 140 (1991).

[31] J. Unguris, R.J. Celotta, D.T. Pierce, J. Appl. Phys. 75, 6437 (1994).

[32] W. Weber, R. Allenspach, A. Bischof, Europhys. Lett. 31, 491 (1995).

[33] D. Stoeffler, F. Gautier, Prog. Theor. Phys. Suppl. 101, 139 (1990); Phys. Rev. B 44, 10389 (1991).

[34] F. Herman, J. Sticht, M. van Schilfgaarde, J. Appl. Phys. 69, 4783 (1991); Mat. Res. Soc. Symp. Proc. 231, 195 (1992).

[35] P. Lang, L. Nordström, R. Zeller, P.H.Dederichs, Phys. Rev. Lelt. 71, 1927 (1993).

[36] S. Krompiewski, F. Süss, U. Krey, Europhys. Lett. 26, 303 (1994).

[37] J. Kudrnovský, V. Drchal, I. Turek, P. Weinberger, Phys. Rev. B 50, 16105 (1994).

[38] Y. Yafet, Phys. Rev. B 36, 3948 (1987).

[39] W. Baltensperger, J.S. Helman, Appl. Phys. Lett. 57, 2954 (1990).

[40] C. Chappert, J.-P. Renard, Europhys. Lett. 15, 553 (1991).

[41] P. Bruno, C. Chappert, Phys. Rev. Lett. 67, 1602 (1991); Phys. Rev. Lett. 67, 2592 (1991) (Erratum); Phys. Rev. B 46, 261 (1992).

[42] R. Coehoorn, Phys. Rev. B 44, 9331 (1991).

[43] J. Barnaś, J. Magn. Magn. Mater. 111, L215 (1992).

[44] R.P. Erickson, K.B. Hathaway, J.R. Cullen, Phys. Rev. B 47, 2626 (1993).

[45] J.C. Slonczewski, J. Magn. Magn. Mater. 126, 374 (1993).

[46] E. Bruno, B.L. Gyorffy, J. Phys., Condens. Mater. 5, 2109 (1993); Phys. Rev. Lett. 71,181 (1993). 
[47] D.M. Edwards, J. Mathon, R.B. Muniz, M.S. Phan, Phys. Rev. Lett. 67, 493 (1991); J. Mathon, M. Villeret, D.M. Edwards, J. Phys., Condens. Mater. 4, 9873 (1992); J. Mathon, M. Villeret, R.B. Muniz, J. d'Albuquerque e Castro, D.M. Edwards, Phys. Rev. Lett. 74, 3696 (1995).

[48] J. d'Albuquerque e Castro, M.S. Ferreira, R.B. Muniz, Phys. Rev. B 49, 16062 (1994).

[49] Y. Wang, P.M. Levy, J.L. Fry, Phys. Rev. Lett. 65, 2732 (1990); Z.P. Shi, P.M. Levy, J.L. Fry, Phys. Rev. Lett. 69, 3678 (1992).

[50] P. Bruno, J. Magn. Magn. Mater. 116, L13 (1992).

[51] M.D. Stiles, Phys. Rev. B 48, 7238 (1993).

[52] J.C. Slonczewski, Phys. Rev. Lett. 67, 3172 (1991); J. Appl. Phys. 73, 5957 (1993); J. Magn. Magn. Mater. 150, 13 (1995).

[53] M.R. Halse, Philos. Trans. R. Soc. London A 265, 507 (1969).

[54] M.T. Johnson, S.T. Purcell, N.W.E. McGee, R. Coehoorn, J. aan de Stegge, W. Hoving, Phys. Rev. Lett. 68, 2688 (1992).

[55] J. Unguris, R.J. Celotta, D.T. Pierce, J. Magn. Magn. Mater. 127, 205 (1993).

[56] A. Fuss, S. Demokritov, P. Grünberg, W. Zinn, J. Magn. Magn. Mater. 103, L221 (1992).

[57] S.N. Okuno, K. Inomata, Phys. Rev. Lett. 70, 1711 (1993).

[58] S.S.P. Parkin, C. Chappert, F. Herman, Europhys. Lett. 24, 71 (1993).

[59] J.-F. Bobo, L. Hennet, M. Piécuch, Europhys. Lett. 24, 139 (1993).

[60] B. Lee, Y.C. Chang, Phys. Rev. B. 52, 3499 (1995).

[61] M.D. Stiles, J. Appl. Phys. 79, 5805 (1996).

[62] P. Bruno, Europhys. Lett. 23, 615 (1993).

[63] P.J.H. Bloemen, M.T. Johnson, M.T.H. van de Vorst, R. Coehoorn, J.J. de Vries, R. Jungblut, J. aan de Stegge, A. Reiders, W.J.M. de Jonge, Phys. Rev. Lett. 72, 764 (1994).

[64] S.N. Okuno, K. Inomata, Phys. Rev. Lett. 72, 1553 (1994).

[65] J.J. de Vries, A.A.P. Schudelaro, R. Jungblut, P.J.H. Bloemen, A. Reinders, J. Kohlhepp, R. Coehoorn, W.J.M. de Jonge, Phys. Rev. Lett. 75, 1306 (1995).

[66] S.N. Okuno, K. Inomata, J. Phys. Soc. Jpn. 64, 3631 (1995).

[67] A. Bounouh, P. Beauvillain, P. Bruno, C. Chappert, R. Mégy, P. Veillet, Europhys. Lett. 33, 315 (1996).

[68] Y. Suzuki, T. Katayama, K. Tanaka, K. Sato, Phys. Rev. Lett. 68, 3355 (1992).

[69] H.S. Bennett, E.A. Stern, Phys. Rev. 137, A448 (1965).

[70] E.N. Economou, Green's Functions in Quantum Physics, Springer-Verlag, Berlin 1983.

[71] R. Kubo, Can. J. Phys. 34, 1274 (1956); J. Phys. Soc. Jpn. 12, 570 (1957); A.D. Greenwood, Proc. Phys. Soc. 71, 585 (1958); Ref. [72], Chap. 4.

[72] R. Kubo, M. Toda, H. Hashitsume, Statistical Physics II: Nonequilibrium Statistical Mechanics, Springer-Verlag, Berlin 1985, Chap. 3.

[73] Y. Suzuki, T. Katayama, A. Thiaville, K. Sato, M. Taninaka, S. Yoshida, J. Magn. Magn. Mater. 121, 539 (1993). 
[74] W. Geerts, Y. Suzuki, T. Katayama, K. Tanaka, K. Ando, S. Yoshida, Phys. Rev. $B$ 50, 12581 (1954).

[75] R. Mégy, A. Bounouh, Y.Suzuki, P. Beauvillain, P. Bruno, C. Chappert, B. Lécuyer, P. Veillet, Phys. Rev. B 51, 5586 (1995).

[76] P. Bruno, Y. Suzuki, C. Chappert, Phys. Rev. B. 53, 9214 (1996). 